\title{
LA RÉUNION ET LE MOUVEMENT DE RÉTROCESSION DE L'ÎLE MAURICE À LA FRANCE (1918-1921)
}

\begin{abstract}
Quiconque a voyagé à travers les colonies des diverses nations ne peut manquer d'établir des différences caractéristiques entre le loyalisme britannique et le patriotisme français ${ }^{1}$.
\end{abstract}

C'est en ces termes que les essayistes réunionnais Marius et Ary Leblond tentent de résumer les différences de principe entre un sentiment d'appartenance spécifique pour la France et une simple allégeance déliée de tout référencement identitaire pour le Royaume-Uni. Dans leur esprit, le loyalisme se comprend avant tout comme fidélité à la Couronne britannique et à ses institutions sans que soit nécessaire un référencement territorial (Home Base $^{2}$ ) et émotionnel (Britishness ${ }^{3}$ ) à l'English Core. En termes politiques, le loyalisme désigne donc l'obéissance des sujets coloniaux britanniques à la Couronne et le respect de ses idéaux. Le respect n'est pas «l'amour » que suppose le patriotisme mais il peut permettre une fidélité sans faille pour qui sait en reconnaitre les vertus en termes de protection et de prospérité.

Cette spécificité permet ainsi au député conservateur mauricien Joseph Adolphe Duclos ${ }^{4}$ d'affirmer qu'une tendance affective attire ses compatriotes vers la France et qu' " une tendance contraire " les dissuade de se séparer, " après plus d'un siècle de coopération loyale de part et d'autre,

1. Marius et Ary Leblond, Exposition coloniale internationale de Paris. L'île de la Réunion, Paris, Société d'éditions géographiques, maritimes et coloniales, 1931, p. 2.

2. Keith Robbins, "The "British Space": World-Empire-Continent-Nation-Region-Locality: A Historiographical Problem ", History Compass, vol. VII, n 1, 2009, p. 66-94.

3. Keith Robbins, "L'historiographie britannique et la "Britishness" ", Revue d'histoire du $\mathrm{XIX}^{\mathrm{e}}$ siècle, $\mathrm{n}^{\circ}$ 37, 2008, (http://rh19.revues.org/index3515.html).

4. Député au Conseil législatif où il représente le district de Flacq, Joseph Adolphe Duclos apparaît comme un précurseur du nationalisme mauricien. 
d'une Angleterre libérale et respectueuse de [leur] profonde francophilie $»^{5}$. A contrario, plus qu'une fidélité politique, le patriotisme colonial français renvoie à un principe identitaire qui relève de l'imaginaire et du symbolique autour d'une mère patrie sublimée. D'ailleurs, Joseph Adolphe Duclos le concède quand il indique que la France que certains Mauriciens aiment n'est autre qu'une "France un peu mystique, la France d'autrefois ${ }^{6} »$. Pourtant, de cette opposition en termes de dévouement et d'allégeance, Marius et Ary Leblond déduisent que les liens entretenus entre la France et ses colonies sont plus profonds et donc voués à perdurer dans le temps. C'est pourquoi, la profondeur des sentiments éprouvés peut servir de modèle aux autres peuples colonisés en quête de reconnaissance identitaire.

Alors que règne cette disposition d'esprit, l'île Maurice est confrontée à une campagne rétrocessioniste entre les années 1918 et $1921^{7}$. Le mouvement est défendu par les Créoles d'origine française qui avancent l'idée d'un retour de l'ancienne "île de France " sous la responsabilité des autorités coloniales françaises. Ces "Franco-Mauriciens ", selon l'expression employée par Hervé de Rauville ${ }^{8}$ pour mentionner les premiers colons de l'île, pensent représenter ce que la "Vraie France " traditionnelle a de meilleur selon une rhétorique très largement éprouvée qui se structure autour d'un « imagined homeland, d'une patrie, d'une langue ancestrale inventée, imaginée $»^{9}$. Le mouvement est certes ponctuel, mais symptomatique d'un contexte colonial en recomposition à la suite des diverses revendications du principe du droit des peuples à disposer d'eux-mêmes, revendications énoncées lors des traités de paix de cet immédiat aprèsguerre. D'aucuns y voient un mouvement identitaire original qui se construit sur une "ancestralité inventée " et autour d'un imaginaire patriotique pro-français.

Dans le même temps, si les Mauriciens se nourrissent des idées assimilatrices ou traditionnalistes françaises pour justifier leurs actions, ces dernières avivent et redynamisent les revendications identitaires et le sentiment d'appartenance nationale des Réunionnais. Ce sentiment peut désormais prendre appui sur une prise de conscience extérieure dont le poids est d'autant plus probant qu'elle se libère des passions politiques internes à La Réunion. Le regard porté sur la France par une frange de la population créole de Maurice permet ainsi aux Réunionnais d'analyser les fondements de leur identité nationale en s'imprégnant de certains lieux p. 6.

5. Jacques Adolphe Duclos, L'Évolution nationale mauricienne, Paris, Jouve \& Cie Éd., 1924,

6. Ibid., p. 391.

7. En termes juridiques, le mot d'origine anglaise se comprend comme la restitution d'un droit acquis. Il s'agit donc de rétrocéder les droits britanniques sur l'île Maurice à la puissance tutélaire originelle : la France.

8. Hervé De Rauville, L'Île de France contemporaine, Paris, Nouvelle librairie nationale, 1908, p. 32.

9. Jocelyn Chan Low, "La France des Rétrocessionistes (1912-1921) ", Revue historique de l'océan Indien, $\mathrm{n}^{\circ}$ 9, 2012, p. 151. 
communs partagés mais également en prenant en compte les spécificités de la situation coloniale mauricienne. En conséquence, cette identification en miroir enrichit les composantes du discours en transférant des sentiments peu éprouvés jusqu'alors en termes d'identité indiaocéanique.

\section{UNE COLONIE DE LA COURONNE EN QUÊTE D'ÉMANCIPATION}

Les origines du mouvement en faveur de la rétrocession s'expliquent par des considérations sociales, politiques et culturelles spécifiques à cette Crown Colony où le pouvoir est entre les mains d'un gouverneur royal omnipotent. En effet, nommé par Londres et dépendant du Colonial office, le gouverneur dispose de compétences élargies même s'il est entouré de conseils consultatifs. Selon John Darwin, la domination autoritaire dans les colonies de la couronne était à l'origine justifiée là où les Britanniques étaient trop peu nombreux pour pouvoir assurer un encadrement politique à tous les échelons de la société10. Effectivement, pour l'île Maurice, il n'y a pas de véritable politique d'implantation de populations originaires du vieux continent pour la période qui nous concerne. Les Anglais y vivent de manière isolée et ne cherchent pas à nouer des contacts avec la population mauricienne qu'ils méprisent globalement sous couvert d'une éthique des races. Cet autoritarisme consubstantiel au statut de la colonie explique en partie l'émergence de certains mouvements d'opposition favorables à une émancipation vis-à-vis de l'autorité de tutelle britannique.

D'un point de vue social et politique, les agitations se sont ainsi multipliées avant-guerre ${ }^{11}$ en raison de nouvelles tensions entre la haute bourgeoisie mauricienne et les membres de "l'intelligentsia créole, de couleur et de petits blancs » pour lesquels il était impossible d'obtenir des emplois à la hauteur de leurs qualifications ${ }^{12}$. Il est vrai que dans cette "société mauricienne verrouillée ${ }^{13}$ ", l'accession aux emplois publics constituait un gage de promotion sociale dans un État colonial que Matthew Lange décrit comme enraciné (embedded), puisque le nombre de fonctionnaires par habitant était un des plus élevés de l'Empire britannique ${ }^{14}$. Cette intelligentsia créole « se sentait souvent humiliée par ce qu'elle considérait comme l'arrogance des riches qui étaient d'avance assurés de la victoire de leurs représentants politiques ${ }^{15}$ ». De surcroit, la Grande guerre a permis un «boom sucrier» faisant la fortune de nombreux oligarques mauriciens

10. John Darwin, The Empire Projects. The Rise and Fall of the British World-System, 1830-1970, Cambridge, Cambridge University Press, 2009, 784 p.

11. Notamment les émeutes raciales de janvier 1911.

12. Sydney Selvon, L'Histoire de Maurice. Des origines à nos jours, Rose-Hill, Mauritius Printing Specialists, 2003, p. 375.

13. Idem.

14. Matthew Lange, "Embedding the Colonial State. A Comparative Historical Analysis of State Building and Broad Based Development in Mauritius ", Social Science History, vol. XXVII, $\mathrm{n}^{\circ} 3$, 2003, p. 397-423.

15. Sydney Selvon, op. cit., p. 377. 
mais creusant par là même les inégalités sociales déjà bien contestées par une frange progressiste de la classe politique mauricienne ${ }^{16}$. Cette précision permet de comprendre la ligne de front établie entre les rétrocessionistes et leurs opposants qui se recrutent pour les premiers parmi les membres de la petite et moyenne bourgeoisie créole, tandis que les seconds se targuent d'appartenir à l'oligarchie blanche, pourtant de vieille souche française et revendiquent une spécificité culturelle que leur francophilie semble justifier.

Ces considérations politiques et sociales se doublent de discriminations raciales exercées à l'encontre des communautés asiatiques installées à Maurice mais aussi envers les Créoles de couleur selon les discours tenus par les partisans de la rétrocession. Les Indiens sont ainsi l'objet de nombreuses attaques dans les médias et deviennent un enjeu de revendications politiques afin de préserver l'identité mauricienne. Selon Édouard Laurent, chroniqueur dans le journal mauricien La Patrie, ses compatriotes ont ainsi à choisir entre deux possibilités : "Rester sujets britanniques et dépendre de l'Inde anglaise, ou redevenir citoyens français et échanger librement [leurs] cerveaux avec tous les peuples de la Terre ${ }^{17}$. " Homme de couleur, ayant fait ses études à La Réunion, Édouard Laurent part ainsi des présupposés raciaux des Britanniques pour réclamer la rétrocession. Il se considère d'abord comme le digne représentant « de l'élément mauricien qui s'est conservé intact depuis $1810^{18}$ " et pense qu'il convient d' " exercer librement les aptitudes que [les Créoles mauriciens tiennent de leurs] ancêtres $^{19}$ ". Cette posture élitaire raciale implique selon ses termes de lutter contre les Indiens qui bénéficient de la mansuétude des Britanniques et dont l'objectif est finalement de noyer les populations d'origine européenne afin de mieux asseoir leur pouvoir ${ }^{20}$. Peu importe l'exactitude d'une telle affirmation, l'argumentation n'a pas alors pour but de défendre une situation conforme à la réalité mais de persuader l'opinion en faisant appel aux sentiments xénophobes d'une frange de la population mauricienne. L'identité se construit ici dans le rejet de l'autre. De manière quelque peu paradoxale, Édouard Laurent dénonce également les discriminations raciales dont les Créoles mauriciens sont victimes. Il considère que la politique coloniale britannique ne peut concevoir l'assimilation des peuples conquis en raison d'une conception racialiste qui prétend expliquer les discriminations sociales par des facteurs héréditaires et raciaux. Prenant d'abord l'exemple de quelques colonies britanniques où la discrimination est de fait reconnue, comme au Cap, Édouard Laurent transpose cette

16. Les rétrocessionistes tel que le $\mathrm{D}^{\mathrm{r}}$ Maurice Curé étaient des socialistes, convaincus du bienfondé des théories de Jaurès notamment.

17. Croix et Patrie, 16 décembre 1919, National Library of Mauritius (NLM), non coté.

18. Idem.

19. Idem.

20. Laurent Édouard, L'Île Maurice entre la France et l'Angleterre, Port-Louis, Impr. de La Patrie, 1913, p. 5. 
situation sur son île en dénonçant l'orgueil ${ }^{21}$ et la condescendance des Anglais en situation coloniale, attitude qui ne peut aboutir qu'à une rivalité fatale. Ainsi, selon lui, «l'Angleterre ne sera et ne pourra jamais être l'amie des races noires, parce qu'un secret instinct lui dit qu'elle devra sa ruine un jour à l'antagonisme qui existe entre ces races et elle $\mathrm{e}^{22}$ ». C'est pourquoi, précise-t-il, l'île Maurice "a été réfractaire à toute assimilation par l'Angleterre, pour la raison qu'il existe une barrière infranchissable entre la race anglo-saxonne et les peuples à peau brune ou de sang-mêlé23 ".

Si la doctrine racialiste britannique dépasse le seul cadre d'un racisme ordinaire, elle alimente les sentiments d'infériorité chez les peuples conquis selon une pression de conformité contre laquelle tentent de lutter les Créoles mauriciens. Selon Édouard Laurent, « le mal le plus grand que nous cause peut-être la domination anglaise, c'est la tare attachée aux hommes de couleur là où flotte le drapeau anglais ${ }^{24}$ ». L'utilisation du mot " tare » entérine à la fois une distinction dégénérative de la société créole de couleur et accrédite l'idée d'une macule à laquelle elle ne peut échapper. C'est pourquoi, poursuit-il, " un assez vif mécontentement règne en ce moment dans la population de Maurice, qui constate avec amertume la réapparition dans leur île de procédés vexatoires qu'ils espéraient abolis pour toujours ${ }^{25}$ ». Qu'importe l'aporie dénonçant le racialisme colonial britannique pour finalement utiliser ses logiques à l'encontre des populations d'origine indienne car l'expression d'une conscience de soi quelque peu mythifiée permet d'utiliser un raisonnement discriminatoire sans se sentir concerné par les conséquences d'une telle logique sur soi-même.

Enfin, le mouvement de rétrocession ne peut se comprendre sans tenir compte des sentiments francophiles d'une grande majorité de la population créole de l'île. De langue et de culture française, l'île Maurice n'a donc pas adopté pleinement la Britishness pourtant garante du loyalisme envers la Couronne. Que ce soit du côté de l'oligarchie ou des classes moyennes, l'unanimité semble de mise sur les vertus supposées de la France et de son modèle colonial considéré comme universel. Les arguments diffèrent cependant en fonction des tendances politiques progressistes ou conservatrices des leaders politiques créoles. Pour les intellectuels conservateurs tels que Hervé de Rauville et Anatole de Boucherville, la France imaginée est surtout celle de l'Ancien Régime, des premiers temps de la colonisation française. Comme le suggère également Joseph-Adolphe Duclos lors d'un discours tenu dans un cercle mauricien à Paris en avril 1919, «la France que nous aimons, n'est-elle pas une France un peu mystique, la

21. Laurent Édouard, L'Île Maurice entre la France et l'Angleterre, op. cit., p. 17.

22. Ibid., p. 10.

23. Aix-en-Provence, Archives Nationales de l'Outre-Mer (dorénavant ANOM) BIB AOM 21354, Émile Appolis, «Un épisode peu connu de l'après-guerre, le mouvement pour la rétrocession de l'île Maurice (1918-1921) ", Actes du 89 congrès national des Sociétés savantes, Lyon, 1965.

24. Laurent Édouard, op. cit.

25. Idem. 
France d'autrefois que nous voyons encore comme à travers un mirage ${ }^{26}$ ?". Si mirage il y a, il s'agit bien d'une illusion, d'une apparence certes séduisante mais trompeuse : un imaginaire francophile, une utopie matricielle qui renvoie à la force d'un nationalisme mystique où les fondements ontologiques de la nation se comprennent à l'aune de l'ancienneté des liens qui unissent les Mauriciens à la France.

Souhaiter que "le jour où rentrera le Roi, pivot de l'harmonie française, il ait le loisir et la puissance de remettre la France dans ses destinées $^{27}$ " relève ainsi d'une vision chimérique peu en phase avec les réalités politiques de la période. Ces désirs témoignent d'une fonction sociale du passé conçu de manière anhistorique où le système politique désiré est totalement en marge des réalités politiques françaises. Cette vision est pourtant créatrice d'un "passé social formalisée ${ }^{28}$ " servant d'alibi pour établir des revendications identitaires. L'ouvrage d'Hervé de Rauville sur L'Île de France contemporaine témoigne notamment de cet ancrage identitaire qui fait fi des bouleversements historiques et du présent colonial ${ }^{29}$. L'auteur nous rappelle que sous le nom d'île de France, Maurice a été une possession française de 1721 à 1810 . Certes, les traités de 1814 et 1815 ont entériné la prise de contrôle britannique. Cependant, au XIX ${ }^{\mathrm{e}}$ siècle, la volonté d'un retour sous le pavillon français s'illustre de manière continue par le désir de certains gouverneurs comme Sir A.-H. Gordon (1871-1874) de léguer l'île aux Français contre un autre comptoir en Inde. De même, Sir Hubert Jerningham, gouverneur de 1892 à 1897 n'hésite pas à affirmer que l'île Maurice « est aussi française aujourd'hui qu'elle l'était alors, peutêtre davantage ${ }^{30}$ ». Émanant de la plus haute autorité coloniale mauricienne, ces déclarations servent de prétexte aux Britanniques pour bloquer tout processus de démocratisation. Le manque de loyauté supposé laisse ainsi penser qu'en cas de conflit avec la France, la francophilie naturelle des Créoles mauriciens les pousserait à trahir la Couronne. Il faut attendre l'Entente cordiale pour que ce point de vue soit quelque peu nuancé de la part des autorités britanniques, malgré les réticences exprimées autour du troisième protocole des accords qui entérinait notamment la possession de la France sur Madagascar.

Pour ces intellectuels conservateurs, l'ensemble de l'argumentation ne débouche cependant pas sur l'idée d'un retour souverain de la France à Maurice, ni même sur l'expression d'une communauté d'intérêts entre l'île Maurice et les possessions françaises du Sud-Ouest de l'océan Indien. L'écueil qui leur semble insurmontable réside dans l'impossibilité d'adapter

26. "M. Duclos à la réunion tenue le 22 avril 1919, à Paris, chez M. Deglos ", in JosephAdolphe Duclos, op. cit., p. 391.

27. Hervé De Rauville, op. cit., p. 345.

28. Éric Hobsbawm \& Terence Ranger (dir.), L'Invention de la tradition, Paris, Éd. Amsterdam, 2012, p. 12.

29. Hervé De Rauville, op. cit.

30. Pierre De Sornay, Isle de France, Ile Maurice, Port-Louis, The General Printing and Stationerey Company Limited, 1950, p. 181-184. 
le régime républicain français à la situation coloniale mauricienne. Si les vertus de la nation française sont reconnues, sa personnification juridique est d'une manière globale rejetée. La République, "aux mains d'un syndicat d'exploiteurs maçonniques et juifs ${ }^{31}$ ", ne peut être l'incarnation politique de la "Vraie France, celle qui travaille et qui prie ${ }^{32}$ ", selon les propos d'Hervé De Rauville. Ayant vécu à Paris durant de nombreuses années et ayant certainement été influencé par la « fièvre hexagonale " provoquée par l'affaire Dreyfus, l'auteur s'inspire, d'une manière indistincte, des diatribes xénophobes d'Édouard Drumont, des publications royalistes de l'Action française et des écrits nationalistes de Jules Lemaitre qui consent d'ailleurs à préfacer son essai ${ }^{33}$. L'adoption du régime républicain remettrait en cause les traditions des « Franco-Mauriciens » et leur foi nationale. L'auteur n'oublie pas de mentionner la perte de "prépondérance ethnique " que provoquerait l'adoption du suffrage universel. Tout en étant dénoncés, ces propos sont repris par Jacques-Adolphe Duclos et témoignent de la force des préjugés en la matière :

En vain quelques esprits chagrins émettent des inquiétudes de Cassandre : "Prenez garde! Le suffrage universel va noyer instantanément la minorité française dans un flot d'Asiatiques et d'Africains. " La masse répond avec Déroulède : «France quand même! " "34.

Le suffrage universel ne servirait donc que de faire-valoir aux prétentions des Asiatiques pour être assimilés. C'est pourquoi le refus d'accéder aux principes républicains est jugé comme un acte de résistance patriotique. En effet, selon Hervé de Rauville, « de toutes les preuves accumulées pour démontrer que la France a totalement dévié de sa voie normale, il n'en est pas de plus décisive que ce spectacle : des Français voulant rester Français et ne pouvant l'être intégralement qu'en répudiant l'union avec la France ${ }^{35}$ ! ». In fine, en se fondant sur un "ailleurs » inventé, un « ailleurs » impossible à accepter, les Franco-Mauriciens dévoilent leur propre crise identitaire et les limites de leur reconnaissance politique au sein d'une société coloniale mauricienne verrouillée.

A contrario, les idéaux patriotiques défendus par Édouard Laurent et ses épigones rétrocessionistes s'inscrivent dans un ancrage républicain qui met tout autant à l'honneur les vertus ancestrales de la nation française mais se repose également sur les valeurs universalistes du régime politique français. Édouard Laurent rappelle ainsi que la République considérée comme " une et indivisible " permet à l'ensemble de ses citoyens d'être égaux en droit quelles que soient leur couleur de peau et leurs conditions sociales. La République est donc appréhendée comme un levier pour une future

31. Hervé De Rauville, op. cit., p. 134-135.

32. Idem.

33. Hervé de Rauville (1858-1935) a été rédacteur pour le Figaro, le Gaulois, La Libre Parole et l'Action française.

34. Joseph-Adolphe Duclos, op. cit., p. 431.

35. Hervé De Rauville, op. cit., p. 134-135. 
émancipation. Il s'agit plutôt d'un nationalisme revendicatif qui, selon les termes de David Bell, peut être défini comme la volonté, jointe à une action politique, de "constituer une communauté politique souveraine regroupant des gens qui ont assez de points en commun - que ce soit la langue, les coutumes, les croyances, les traditions ou autres - pour leur permettre d'agir comme une individualité homogène et collective ${ }^{36}$ ". Il s'agit principalement d'une vision héritée des Lumières faisant de la nation une notion juridique à part entière dans une acception révolutionnaire et établissant le principe de l'égalité des citoyens devant la loi. Le principe de la mission universelle est dès lors associé à celui de la mission civilisatrice pour garantir le progrès de l'humanité, selon les propos tenus par Abel Loumeau dans le journal Croix et Patrie :

"France! Avant-garde de la Civilisation à l'assaut de l'ignorance, pionnier du progrès, c'est toujours vers toi que l'humanité se tourne pour connaitre sa voie dans la marche évolutive vers ses destinées ${ }^{37}$ ".

Finalement, cette vision ne se démarque guère du référencement chronologique des Franco-Mauriciens conservateurs qui établissent eux aussi leur idéal national à partir des débats de la pensée contre-révolutionnaire. L'exaltation nationale autour d'une France républicaine est ainsi comprise comme un moyen de s'émanciper non seulement de la tutelle oppressive du Royaume-Uni mais également de la pesanteur coloniale mauricienne maintenue par une oligarchie blanche soucieuse de garantir ses intérêts. C'est sur ce terreau identitaire que se greffent les mouvements politiques en faveur d'une rétrocession de l'île Maurice à la France. Si la campagne d'opinion bat son plein au lendemain de la Grande Guerre, certains signes avant-coureurs sont néanmoins décelables lors du déclenchement des hostilités.

\section{LES PRÉMICES D’UN MOUVEMENT FRANCOPHILE}

Le mouvement rétrocessioniste ne peut se comprendre sans une analyse du contexte diplomatique et une mise en perspective de certains épisodes vécus conjointement par les Mauriciens et les Réunionnais. Les signes avant-coureurs sont ainsi à chercher dans deux événements précédant la campagne rétrocessioniste qui permettent de comprendre certaines permanences dans les discours. Que ce soit les effusions patriotiques lors de l'entrée en guerre en 1914 ou les célébrations de la Victoire en 1918, chaque épisode témoigne d'une politisation accrue des débats identitaires.

Les manifestations patriotiques observées à l'île Maurice lors de l'entrée en guerre en 1914 témoignent de sentiments ambigus vis-à-vis de la France et de l'Angleterre. La population mauricienne est informée du

36. David Bell, The Cult of the Nation in France. Inventing nationalism, 1680-1800, Cambridge, Harvard University Press, 2001, p. 20.

37. Croix et Patrie, 31 octobre 1919, NLM, non coté. 
déclenchement du conflit dès le 5 août 1914. Les manifestations officielles sont suivies devant l'Hôtel du Gouvernement à Port-Louis par une «foule immense, où toutes les classes de la communauté " sont représentées, selon les propos du journal mauricien Le Cernéen ${ }^{38}$. Les discours des élites politiques se succèdent avec une grande spontanéité même s'il s'agit surtout de prouver son loyalisme et donc son allégeance aux autorités britanniques. De manière entendue, les journaux comparent les propos tenus par les loyalistes britanniques et les francophiles rétrocessionistes. Pour les loyalistes, l'enthousiasme de la foule met en évidence une communauté d'action et de pensée entre les aspirations patriotiques de l'opinion publique mauricienne et les directives émanant des autorités coloniales. Le gouverneur de l'île Maurice, John Middleton, salue cette "magnifique manifestation de loyalisme ${ }^{39}$ " et ce même sentiment d'amour que les Mauriciens éprouvent vis-à-vis de l'Angleterre et de la France. À l'unisson, le God save the King et la Marseillaise sont chantés par une foule unie et solidaire malgré les «obligations qui paraissent pénibles au premier abord " et le devoir patriotique attendu pour chaque membre des communautés de l'île.

Ce sentiment d'un devoir à accomplir se comprend dans un double élan de loyauté. Il s'agit à la fois d'obéir aux injonctions des autorités britanniques et de respecter la promesse d'une solidarité entre la France et l'Angleterre selon les termes des accords de l'Entente Cordiale. Honneur et devoir se conjuguent pour imposer une morale patriotique sans faille. Le gouverneur Middleton ne s'y trompe pas lorsqu'il rappelle les propos tenus par le Foreign Secretary, Sir Edouard Grey, devant la chambre des Communes à la veille de l'entrée en guerre. Celui-ci parlait alors " des liens d'amitié unissant les deux grandes nations et de l'appui que la Grande-Bretagne devait apporter à la France ${ }^{40}$ ». La foule se rendit ensuite au consulat de France, où elle chanta de nouveau les deux hymnes nationaux et acclama le consul qui, "profondément ému, remercia vivement les manifestants, leur donnant rendez-vous à la première victoire remportée par les armes françaises ". Non sans ambiguité, le journal termine son compte rendu de la manifestation en rappelant que " chacun s'est senti fier d'appartenir au glorieux drapeau pour la défense duquel tous les fils de Maurice sont prêts à verser la dernière goutte de leur sang ${ }^{41}$ ! ». La couleur de ce drapeau, si fièrement brandi, n'est cependant pas précisée, laissant aux lecteurs du journal la liberté d'en dessiner les contours. Dans un contexte d'union et de nécessaire apaisement politique, les propos tenus par Le Cernéen s'inscrivent donc dans une volonté d'ouverture plutôt francophile. Pourtant loyaliste et opposé aux thèses rétrocessionistes, le journal tente d'initier un rapprochement de circonstance que les conditions du conflit semblent imposer.

38. Le Cernéen, 6 août 1914, NLM, non coté.

39. Idem.

40. Le Cernéen, 6 août 1914, op. cit.

41. Idem. 
A contrario, lorsque le journal rétrocessioniste Le Mauricien publie son compte rendu des manifestations, son lectorat peut s'attendre a priori à une émouvante déclaration de soutien en faveur de la France. Pourtant, sous la plume d'Eugène Henry, le journal témoigne d'une relative prudence qui se transforme très vite en une volte-face politique sans doute surprenante pour un lectorat francophile habitué aux diatribes contre les infortunes de l'administration britannique. En effet, selon Eugène Henry, "les cœurs des Mauriciens battent à l'unisson de ceux des Anglais et des sujets britanniques du monde entier. Ils ont confiance dans le triomphe de la noble cause que défend l'Angleterre et espèrent bientôt apprendre que la flotte britannique a anéanti celle de l'Allemagne ${ }^{42}$ ". C'est pourquoi, poursuit-il, les Mauriciens ont tenu à prouver " encore une fois que leur fidélité à la Couronne britannique n'est pas un vain mot et que, lorsque la mère-patrie est en danger, ils tiennent à participer à sa défense ". Que le loyalisme soit clairement exprimé n'a rien de surprenant. Cependant, que l'on se réfere à une mère-patrie qui n'est pas encore véritablement en danger devait laisser pantois un lectorat peu accoutumé à une telle magnanimité envers les autorités britanniques. S'agit-il finalement d'une allusion à la France, véritable mère-patrie que le journal encense habituellement ? De la France " en danger " si l'on se réfere aux discours prenant réellement en compte le contexte de la guerre ? Aucune réponse n'est véritablement apportée par le compte rendu de la manifestation. C'est peut-être pourquoi, deux jours plus tard, le journal tient à préciser sa pensée en rappelant que les Mauriciens sont « intimement liés à deux des grandes nations alliées, autant par les liens du sang que par loyalisme et reconnaissance pour les bienfaits qu'ils en ont reçus ${ }^{43}$ ». Les sentiments éprouvés "pour la France et l'Angleterre sont trop vivaces et inspirés par l'affection la plus pure ${ }^{44}$ » pour que le journal puisse tenir un autre langage. Nous pouvons ainsi émettre l'hypothèse d'un loyalisme de circonstance permettant aux rétrocessionistes de laisser de côté leur francophilie exclusive et radicale d'avant-guerre pour mieux asseoir la légitimité politique de la communauté créole de couleur. En faisant un tel acte d'allégeance, les rétrocessionistes espéraient sûrement attirer la bienveillance des autorités britanniques sur leur devenir politique. C'est pourquoi, au lendemain de la guerre, les débats reprennent avec d'autant plus d'acuité, de sensibilité et de clairvoyance que nul ne peut être accusé d'avoir dérogé à la règle de la fidélité.

En 1918, les célébrations de la victoire sur l'île Maurice mettent surtout en évidence un loyalisme sans faille à la Couronne britannique. Dès que les conditions de l'armistice sont connues, les autorités mauriciennes organisent des manifestations patriotiques au cours desquelles sont célébrés l'Angleterre et ses alliés. La Marseillaise est entonnée de concert avec le God Save the King sans que cela ne pose de problème de référencement 
patriotique. Les édiles mauriciens s'empressent d'exprimer leurs sentiments de fidélité qui sont très largement diffusés par la suite dans les médias de l'île. C'est ainsi qu'Armand Esnouf, député du district de Plaines Willems, met en avant le sens de l'honneur des Britanniques qui n'ont pas hésité en 1914 à voler au secours de la France. L'Angleterre ayant mis «son épée au service du droit et de la justice ${ }^{45}$ ", selon les termes employés par le gouverneur Hesketh, a rempli «d'une patriotique fierté le cœur des peuples de l'Empire ${ }^{46}$ ». Le conseil de gouvernement renchérit le 26 novembre 1918 par l'intermédiaire d'Henry Leclézio en proclamant solennellement "l'expression de sa loyauté et de son attachement à l'Empire, ainsi que sa fierté de faire partie de cet Empire ${ }^{47}$ ". Seul le $\mathrm{D}^{\mathrm{r}}$ Eugène Laurent ose revendiquer pour les Mauriciens l'accession au statut de citoyen britannique en guise de reconnaissance des bons et loyaux services rendus à la Couronne. Cette reconnaissance serait, selon ses termes, une juste récompense de "notre loyauté, notre dévouement et notre attachement au Roi et au trône britannique ${ }^{48} »$.

Les espoirs des Mauriciens vont très vite être déçus lorsqu'ils vont constater que le loyalisme dont ils ont témoigné avec force n'a aucunement infléchi la politique de la Couronne. Dès le mois de novembre 1918, les premiers signes d'un désenchantement se font jour et relancent la problématique identitaire comme en témoigne cet éditorial du journal Le Mauricien :

Il n'est pas possible que la France, notre patrie d'origine, l'Angleterre, notre patrie d'adoption, ne s'entendent pas pour faire cesser le douloureux dilemme où nous nous débattons : d'être restés trop Français pour être considérés comme Anglais, et d'être trop sujets britanniques pour être considérés comme Français ${ }^{49}$.

Certes, cette non-reconnaissance des droits politiques et la frustration qu'elle engendre sont partagées par l'ensemble des populations colonisées des deux empires. Cependant, la situation mauricienne montre une certaine singularité dans le sens où les revendications émancipatrices y étaient déjà prégnantes bien avant l'ouverture des hostilités. C'est peut-être cette non-reconnaissance qui explique par la suite la virulence des débats autour du projet de rétrocession. Les deux puissances coloniales victorieuses sont alors présentées comme deux modèles contrastés d'intégration coloniale. Si le Royaume-Uni est perçu comme un modèle d'intégration élitaire où seuls les oligarques sucriers peuvent tirer leur épingle du jeu, la France est imaginée comme un modèle d'intégration plus universel où les Créoles de couleur peuvent espérer une reconnaissance plus élargie de leurs droits.

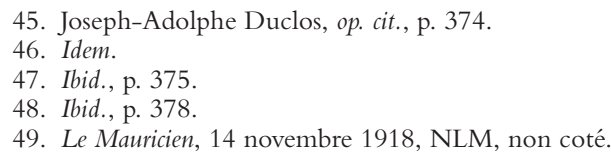




\section{LA CAMPAGNE DE RÉTROCESSION DE L'ÎLE MAURICE À LA FRANCE}

Durant la Grande Guerre, les débats sur la rétrocession ont été étouffés pour préserver les efforts de guerre entrepris par les deux puissances coloniales et garantir une paix politique locale. C'est pourquoi les revendications ne s'expriment de nouveau avec force qu'au lendemain de l'armistice. De nombreux intellectuels mauriciens entrent dans le débat et développent une argumentation qui tient compte à la fois des fondements identitaires initiaux mais également de nouveaux principes émanant du nouvel ordre international. De surcroît, les enjeux dépassent le contexte indiaocéanique pour désormais s'inscrire dans le domaine impérial des deux puissances coloniales britannique et française.

Le mouvement favorable à la rétrocession se structure peu à peu autour de meetings politiques qui célèbrent l'héritage français sur l'île Maurice. C'est ainsi que, le $1^{\text {er }}$ février 1919, devant l'Hôtel de ville de Port-Louis, un banquet est organisé en l'honneur de la France. Les militants rivalisent de déclarations emphatiques sur ses vertus et les bienfaits de son héritage. Tel est le cas du discours prononcé par le $\mathrm{D}^{\mathrm{r}}$ Maurice Curé qui déclare aimer la France pour «les liens du passé, la beauté resplendissante de son histoire, les forces morales qu'elle représente ${ }^{50} »$. Le poète Léoville L'Homme rappelle également que "la France nous avait préparé un trésor de bienfaits qu'il a été doux à nos pères de nous transmettre ${ }^{51} »$. Ce référencement national tient donc plus d'une culture et de valeurs partagées, d'un héritage en commun que des liens du sang. Le 5 mars 1919, un autre meeting est organisé au théâtre de Port-Louis. Ce dernier fonde l'action du mouvement au sens où un organigramme de ses responsables est proposé ainsi qu'un programme d'actions qui dépasse le seul cadre mauricien. Présidé par Anatole de Boucherville, pourtant réticent aux idées rétrocessionistes avant-guerre, le meeting réunit plus de trois cents participants choisis parmi les " représentants des classes les plus éclairées de la population mauricienne ${ }^{52}$ ". Lors du meeting, des représentants mauriciens vivant à Paris sont désignés afin d'être au plus près des instances gouvernementales françaises. Paul Carié, Joseph Rivière, le $\mathrm{D}^{\mathrm{r}}$ Baschet, Loïs Dabbadie, Davis Edwards, Ange Galdemar et Hervé de Rauville sont ainsi nommés pour structurer un lobby rétrocessioniste dans la capitale française. La constitution de ce comité parisien est le fruit d'un compromis politique puisque certains de ses membres étaient préalablement opposés à la rétrocession. Cette ouverture n'est pas anodine si nous considérons que, par ce biais, les revendications de certains Franco-Mauriciens rejoignent désormais celles des Créoles de couleur de l'île. Cette union de circonstance, structurée autour d'une francophilie partagée bien que comprise différemment, permet de

50. Banquet en l'honneur de la France victorieuse, $1^{\text {er }}$ février 1919, The General Printing and Stationerey Company Limited, Port-Louis, 1919, III, 31 p. NLM, non coté.

51. Idem.

52. Croix et Patrie, 6 mars 1919, NLM, non coté. 
donner plus de poids politique au mouvement. Libérés de toute contrainte communautaire, les rétrocessionistes élargissent leur champ d'action aux structures gouvernementales centrales des deux puissances coloniales.

Le 4 avril 1919, les représentants parisiens adressent ainsi une requête en faveur de la rétrocession à Lloyd George et à Georges Clemenceau. Selon leurs propos, la rétrocession à la France se justifie « au nom du droit des peuples de disposer d'eux-mêmes ${ }^{53}$ ". C'est donc en termes juridiques que les motivations sont d'abord exprimées puisqu'elles défendent une certaine conception de la citoyenneté, de la nation et du droit international. Certainement influencés par les comptes rendus des conférences de paix qui se déroulent au même moment, les responsables du comité font preuve d'un pragmatisme politique qui les éloigne quelque peu des principes identitaires défendus initialement. Leur argumentation reprend également les principes d'une convergence d'intérêts politiques et économiques entre La Réunion, Madagascar et l'île Maurice. Sans évoquer explicitement le principe d'une identité commune pour cette union des peuples indiaocéaniques, les représentants du mouvement se réferent à la fois à la politique des " groupements naturels $s^{54}$ " dans le monde colonial et aux principes wilsoniens du droit des peuples à disposer d'eux-mêmes. Ils pensent également que pour asseoir une paix durable, il conviendrait de délimiter des sphères d'influence plus précises et plus clairement circonscrites à des aires coloniales "naturelles ${ }^{55}$ ". Le contexte leur semble favorable compte tenu de l'expression d'une solidarité sans faille durant le conflit. En réclamant la légitimité de certains droits et en s'inscrivant dans une volonté de pacification des relations franco-britanniques, les membres du comité espèrent susciter un intérêt des instances exécutives centrales.

Sur l'île Maurice, le mouvement se structure également de manière plus précise autour du $\mathrm{D}^{\mathrm{r}}$ Maurice Curé, des journalistes Raoul Rivet et Eugène Henry du journal Le Mauricien mais aussi d'Anatole de Boucherville alors rédacteur en chef du quotidien Croix et Patrie. Les débats relayés dans la presse incitent les hommes politiques de l'île à se positionner sur cette question. Édouard Laurent contacte à cet effet Henry Leclézio dans une lettre en date du 24 février 1919, où il lui demande de "rendre gloire à la France, le berceau de [ses] ancêtres ${ }^{56}$ ». Pour toute réponse, le doyen du Conseil législatif se contente d'expliquer pourquoi il reste attaché à l'Angleterre et continue dans le même temps à aimer la France, réponse on ne peut plus diplomatique et stratégique.

Les opposants au projet comprennent dès lors l'urgence d'une structuration de leur action. Ils se regroupent autour du leadership de JosephAdolphe Duclos alors député au Conseil législatif et surtout réputé pour le pragmatisme politique que lui conferre sa formation initiale de juriste. 
Ce dernier s'embarque pour l'Europe et organise le 22 avril 1919 à Paris une réunion avec des militants opposés à la rétrocession. Prenant appui sur un comité étoffé, il les convainc de signer une résolution destinée à Lloyd George qui, tout en prônant un loyalisme envers les autorités britanniques, va plutôt dans le sens d'un apaisement.

La réponse au comité faite par le secrétaire d'État des Colonies britanniques insiste sur la volonté royale de maintenir le statu quo et de ne pas entrer dans la polémique ${ }^{57}$. Malgré cette ligne de conduite officielle, certains fonctionnaires du Foreign office sont pourtant favorables à la rétrocession. Tel est le cas de Sir Francis H. Skrine qui publie dans le Times un article pour le moins iconoclaste par rapport à la politique officielle de son ministère. Il estime en effet " que l'avenir de l'île Maurice devrait attirer l'attention de la Conférence de la Paix " et qu'en " dépit d'une longue occupation britannique, l'île Maurice est une île française ! " ${ }^{58}$. Joseph-Adolphe Duclos s'indigne de cette prise de position et répond dans le même journal par un argumentaire expliquant la nécessité pour le Royaume-Uni d'envisager l'île Maurice comme un point stratégique essentiel pour l'océan Indien. Cependant, au-delà des intérêts britanniques, Joseph-Adolphe Duclos insiste surtout sur la volonté des Mauriciens qui dans son esprit refusent totalement le principe d'une rétrocession malgré les liens qu'ils entretiennent avec la France.

Les débats sont alors relayés dans la presse britannique qui se fait fort de rappeler l'action pernicieuse menée par certains publicistes et hommes politiques français. Le Times réagit ainsi à un discours au Sénat français d'Eugène Réveillaud qui compare la situation de Maurice à celle de l'Alsace et de la Lorraine, une "Alsace-Lorraine du grand océan " en quelque sorte. Son objectif est de restituer au «patrimoine de la France tout ce qui en a été détaché au temps [des] revers nationaux ${ }^{59}$ ". Le Times reprend les arguments de Joseph-Adolphe Duclos lorsque ce dernier indique que les rétrocessionistes ne "reflètent d'aucune façon les sentiments de la population de l'île ${ }^{60} »$. Le journal n'y voit que l'excitation stérile d'une opinion française manipulée par un groupe " restreint mais très actif de Mauriciens ${ }^{61} "$. La comparaison établie entre la situation de l'île Maurice et celle de l'Alsace-Lorraine désireuse de retrouver le giron de sa mère-patrie démontre, selon le journal, " la part d'ignorance dans l'idée qu'on se fait en France de cette question ${ }^{62}$ ». Surtout, le journal y voit un nouveau prétexte pour exposer des griefs contre le Royaume-Uni afin de "détruire l'efficacité de l'amitié franco-britannique ${ }^{63}$ ". Même si cette agitation rétrocessioniste ne présente aucun danger, elle est condamnable

57. Ibid., p. 399.

58. Pierre De Sornay, op. cit., p. 184.

59. Émile Appolis, op. cit.

60. ANOM 1AFFPOLC58, The Times, 10 mars 1920.

61. Idem.

62. Idem.

63. Idem. 
car elle permet à certains hommes politiques français de se saisir de « la moindre occasion de [...] faire une guerre de coups d'épingle ${ }^{64}$ ".

Dans le même temps sur l'île Maurice, les partisans de la rétrocession accentuent leur action et font publier une série de brochures. Après la publication par la délégation mauricienne de Paris d'un essai résumant les grandes idées rétrocessionistes ${ }^{65}$, de nombreux militants profitent de la moindre tribune pour exposer leur point de vue. Tel est le cas notamment de Paul Carié, dont la conférence à la Société de Géographie de Paris le 9 janvier 1920, est relayée dans les médias colonialistes français. Tel est surtout le cas de Joseph Rivière qui écrit en 1920 une brochure intitulée L'île de France à la France ${ }^{66}$. Francophile ardent, il est d'ailleurs fait chevalier de la Légion d'Honneur dès 1903 avant d'obtenir le grade d'officier en 1914 pour son implication au sein de l'Association médicale franco-anglaise. Ayant vécu à La Réunion durant de nombreuses années, il témoigne de la générosité de la France en faveur des Réunionnais notamment dans le domaine de l'instruction. Selon ses termes, la rétrocession permettrait de mettre fin à une période de "barbarie » et de "violence " :

Serait-il admissible que la Société des Nations pût autoriser l'étonnant spectacle d'une colonie, française par les traditions, les mœurs et le langage, qui ne serait pas gouvernée par des Français ${ }^{67}$ ?

Se faisant le relais de l'ensemble de ces brochures, les médias français radicalisent la campagne d'opinion en faveur de la rétrocession. Que ce soient Le Journal, Le Cri de Marseille, Actualités, Le Moniteur de la Flotte, le Courrier Saïgonnais ou le Courier Colonial tous ces journaux semblent unanimes pour en défendre la cause. Le mouvement n'est pourtant guère suivi par la classe politique française à de rares exceptions près.

C'est dans ce climat que surviennent à Maurice les élections de janvier 1921, que les circonstances vont transformer en une sorte de référendum favorable ou opposé à la rétrocession ${ }^{68}$. L'un des candidats favorables à la rétrocession, Armand Esnouf adresse ainsi un manifeste à ses partisans le 8 janvier 1921 :

Tout en rendant un juste hommage à l'Angleterre pour la façon dont elle nous a traités, n'ai-je pas le droit de dire, et de dire bien haut, dans mon pays, chez moi en somme : mes ancêtres étaient français, mon pays était français, ma patrie est la France, l'Angleterre elle-même me dénie le droit d'être citoyen anglais, je veux retourner à mes origines ${ }^{69}$.

64. Idem.

65. Le retour de l'île Maurice à la France. Documents publiés par la délégation mauricienne de Paris, Paris, Les Imprimeries J. Brard, 1919, 67 p.

66. Joseph Rivière, L'Île de France à la France, Paris, Philippe Renouart, 1920, 14 p. NLM, non coté.

67. Idem.

68. En vertu des réformes de 1885 qui introduisent le principe électif dans la constitution coloniale mauricienne, des élections au suffrage censitaire sont régulièrement organisées pour constituer en partie le Conseil du gouvernement. Les membres «nommés " par le gouverneur restent pourtant majoritaires au sein de ce conseil.

69. Jacques-Adolphe Duclos, op. cit., p. 469. 
Les élections provoquent donc une ligne de partage au sein de la société mauricienne. D'une manière globale, les Franco-Mauriciens les plus riches et les sujets d'origine britannique dans leur grande majorité sont opposés au rattachement. Les populations de couleur d'origine française y sont plus favorables et témoignent par cet engagement de leurs réticences envers les préjugés raciaux des autorités britanniques. Les Indiens quant à eux y sont résolument opposés même si certains de leurs représentants ont pu à un moment donné se laisser tenter par un espoir d'émancipation en adoptant une position favorable aux rétrocessionistes. Le gouvernement anglais se défend d'intervenir dans les combats électoraux. Pourtant, pour prévenir des troubles éventuels, l'Amirauté envoie en rade de Port-Louis un navire de guerre, le Comus afin d'assurer le déroulement " pacifique " des élections.

Dans tous les districts, les résultats s'avèrent entièrement défavorables aux rétrocessionistes puisque globalement leurs adversaires totalisent près de $72 \%$ des suffrages. Les élections semblent avoir tranché la question. C'est bien ainsi que l'entend le gouvernorat britannique. Le 31 mai 1921, en ouvrant la session du nouveau Conseil législatif, le gouverneur de Maurice rappelle que

par une énorme majorité, l'électorat a donné une preuve écrasante de son entier attachement à l'Empire, et il est absolument clair que l'inextinguible dévotion des vieilles familles de Maurice à la terre bien-aimée de leurs ancêtres est parfaitement compatible avec leur loyalisme envers le glorieux drapeau qui les abrite depuis plus de cent ans ${ }^{70}$.

L'agitation médiatique autour du projet de rétrocession est relayée dans les milieux diplomatiques français et dans la presse réunionnaise. Les enjeux sont d'autant plus importants qu'il s'agit d'apaiser les tensions entre le Royaume-Uni et la France mais aussi de prendre en compte les revendications réunionnaises sur ce sujet. Dans le contexte de l'immédiat aprèsguerre, les principes défendus par les rétrocessionistes permettent ainsi de catalyser de nouvelles formes d'expression identitaire autour des liens qui unissent la France à ses colonies indiaocéaniques.

\section{PRISE DE CONSCIENCE D’UNE IDENTITÉ INDIAOCÉANIQUE}

La correspondance du vice-consul de France à Port-Louis, M. Girieud, permet d'entrevoir une analyse en miroir de l'agitation politique provoquée par le mouvement rétrocessioniste. L'ensemble de ses rapports met en évidence à la fois l'embarras des autorités françaises et le jeu de dupes qui s'échafaude peu à peu.

En effet, ne souhaitant aucunement se mettre à dos la communauté francophile mauricienne et une opinion réunionnaise de plus en plus 
favorable aux thèses rétrocessionistes, l'autorité consulaire affiche une complaisance de façade tout en jugeant que "ce mouvement fait dans un but impossible, ne pourrait produire que dissensions et désordres ${ }^{71}$ ". Certes, le mouvement soulève " dans le public des commentaires, mais la presse locale, certainement retenue par un mot d'ordre du gouvernement, reste silencieuse ${ }^{72}$ ». De la sorte, le vice-consul fait échouer toutes les démarches entreprises par les rétrocessionistes auprès du consulat afin de ne pas impliquer la diplomatie française.

La correspondance établie avec le ministère des Affaires étrangères montre que la situation est perçue avec une certaine gravité puisque certains parlementaires français interviennent en faveur de la rétrocession et que le ministère des Colonies par le biais des informations fournies par le gouverneur de La Réunion est lui-même impliqué. Les informations transmises sont parfois confuses et démontrent que la question est d'autant plus complexe que les pourparlers de paix à Versailles nécessitent une communion de pensée entre le Royaume-Uni et la France. Dans le même temps, les groupes de pression s'activent en France et soutiennent le principe d'une rétrocession. Certaines municipalités appuient d'ailleurs cette demande comme celle de Tarbes qui est la plus prompte à le faire, le 3 février 1920. D'autres communes comme Saint-Malo ou Lorient font de même par la suite. Le conseil de la Société des Études Coloniales et Maritimes, réuni sous la présidence du vice-amiral Besson, enjoint également le gouvernement français à obtenir du gouvernement britannique la rétrocession à la France de l'île Maurice avec les îles de Rodrigues et des Seychelles. L'argumentation avancée est celle de la complémentarité économique de ces territoires. Il en est de même de la Ligue coloniale française dans sa séance du 17 décembre 1919 et de la Chambre de Commerce de Marseille dans sa séance du 6 mai $1919^{73}$. Ce lobbying pro-rétrocessioniste est notamment soutenu par le journal La Presse coloniale dont le directeur est le député réunionnais Georges Boussenot.

La presse réunionnaise se lance également dans les débats et établit d'emblée des comparaisons entre les deux systèmes coloniaux défendus par les puissances française et britannique. L'opinion est depuis longtemps sensibilisée aux thèses développées par le mouvement rétrocessioniste car la presse réunionnaise a régulièrement rendu compte des conférences et des écrits d'Édouard Laurent. Même si au début du mouvement les journaux se sont montrés prudents en ne commentant que de manière allusive ses propos, ils défendent ensuite avec emphase son action et interprètent la rétrocession comme un juste retour aux droits historiques de la France. Dans le même temps, les critiques énoncées à l'encontre des autorités mauriciennes se comprennent à l'aune des revendications assimilatrices de

71. ANOM 1AFFPOLC58, Vice-consul de France à Port-Louis au président du Conseil, 25 juillet 1920 ,

72. ANOM 1AFFPOLC58, Vice-consul au ministre des Affaires étrangères, 29 janvier 1919.

73. ANOM 1AFFPOLC58, La Presse coloniale, 15 mai 1919. 
La Réunion et du rejet des populations indésirables qui la composent. Rétrocession, assimilation et xénophobie sont donc les points d'ancrage d'une même base argumentative où se confondent la revendication de droits et la défense d'une identité spécifique.

Les premiers commentaires explicites sur la rétrocession datent de mars 1918. Le journal réunionnais Le Peuple considère ainsi que " tous les Anglais, suffisamment honnêtes pour voir clair et reconnaître le patriotisme des Mauriciens envers la France, savent qu'un échange de territoires est l'immanquable solution ${ }^{74}$ ". La France, grâce à sa politique d'assimilation, peut favoriser l'émancipation des Mauriciens confrontés à la politique d'exclusion ou de "noyage des ethnies » menée par les Britanniques. En ce sens, selon le journal, les Mauriciens ne peuvent ressentir des sentiments patriotiques envers la Grande-Bretagne puisqu'ils ne peuvent espérer acquérir des droits et se trouvent confrontés à une mixité ethnique peu propice à la construction d'une identité respectueuse de leurs origines. Comme le souligne encore Le Peuple, le Royaume-Uni aurait tout intérêt à se délester d'une colonie qui " n'a plus de valeur stratégique ${ }^{75}$ " et dont les troupes pourraient être employées ailleurs. Ce choix parait d'autant plus impérieux que "la population est difficile à gouverner et ne sera jamais anglicanisée $e^{76}$ ". Le journal avance donc l'idée d'échanger ce territoire contre la Nouvelle-Calédonie ou les Nouvelles-Hébrides, ce qui permettrait à la France de maitriser l'océan Indien. Le journaliste pense que l'opinion favorable aux rétrocessionistes donne aux Réunionnais

un peu plus de fierté de ce [qu'ils sont], car on avait trop voulu [les] rabaisser, il y a quelques années, en faveur de Maurice. Elle peut donc contribuer à [les] rendre plus heureux en [leur] ouvrant les yeux sur [leur] bonheur ${ }^{77}$.

Le mouvement de rétrocession aboutit finalement à la construction d'un imaginaire indiaocéanique où le rattachement de La Réunion à Maurice et éventuellement à Madagascar permettrait l'expression d'une identité commune car comme le rappelle Joseph Rivière, "la guerre mondiale a démontré à tous les esprits clairvoyants la nécessité de restaurer les groupements ethniques naturels ${ }^{78} "$.

Les deux îles se complètent et, avec Madagascar, forment, suivant l'expression de la Ligue coloniale française, un tout indivisible. [...] Elles pourraient, si Maurice nous était rendue, constituer dans la mer des Indes un bloc français ${ }^{79}$

Comme le souligne la presse réunionnaise, un échange de souveraineté est depuis longtemps envisagé du côté mauricien. C'est pourquoi,

74. Archives départementales de La Réunion (dorénavant ADR) 1PER81/24, Le Peuple, 23 mars 1918.

75. ADR 1PER81/24, Le Peuple, 11 avril 1918.

76. Idem.

77. Idem.

78. Joseph Rivière, op. cit.

79. Paul Carié, Société de Géographie de Paris, 9 janvier 1920, op. cit. 
toujours selon Le Peuple, "l'intérêt nous commande de les aider à se libérer du joug étranger ${ }^{80}$ ". Se faisant l'écho du journal mauricien Croix et Patrie, les journaux réunionnais reprennent à leur compte cette idée que « le droit découle de faits historiques, d'actes antérieurs, à l'actif de celui qui veut aujourd'hui agir ${ }^{81}$ ». La seule justification à l'action présente ou future gît donc dans l'action passée. Là est le droit,

et nul ne peut venir dire "ôte-toi de là que je m'y mette" à moins de démontrer que celui qui y est a démérité, qu'il doive céder la place et être dépossédé d'un droit qu'il a mais qu'il n'est plus digne d'exercer ${ }^{82}$.

Cette idée d'associer le destin de La Réunion à celui des îles voisines est ancienne et procède de logiques de recompositions coloniales d'avantguerre.

Cependant, cette identité indiaocéanique qui se construit sur des aspirations communes repose en partie sur l'exclusion des Asiatiques. Selon Raphaël Barquissau,

dans l'île voisine, Maurice, l'intransigeance subsiste dans toute sa rigueur, encouragée par la morgue anglo-saxonne à l'égard des coloured people ; mais l'indianisation du pays y guette les colons blancs, sournoisement favorisée par une certaine politique anglaise pour triompher des vieilles résistances françaises à la domination des British Born ${ }^{83}$.

Pour lutter contre cet envahissement asiatique qui menace tout autant La Réunion que Maurice, la solution qu'il envisage serait d'organiser une émigration massive à Madagascar des Créoles des deux îles. Dans la presse coloniale, les discours sont identiques. Si les Anglais ont bel et bien su trouver un compromis politique à Maurice, en accordant au début de leur prise de possession des postes à des Mauriciens et en respectant leurs coutumes, l'abolition de l'esclavage a toutefois provoqué une crise " et l'Angleterre n'ayant pu s'assimiler ses nouveaux sujets par la douceur, prétendit se les assimiler par la force ${ }^{84}$ ». Les Mauriciens ont alors dû lutter contre "leurs maîtres qui affichaient ouvertement leur intention de faire de l'ancienne île de France, l'île d'Angleterre, en forçant les Franco-Mauriciens à s'expatrier et à céder leurs biens à des citoyens du Royaume-Uni ». Cette politique se concluant par un échec, "l'Angleterre voulut les noyer dans un flot d'Hindous ». De surcroît, en nommant un "Hindou " au poste de gouverneur en $1883^{85}$ et en accordant soi-disant aux Indiens de nombreux emplois administratifs, ces derniers ont pris peu à peu possession

80. ADR 1PER 81/24, Le Peuple, 6 mai 1918.

81. ANOM BIB SOMf/POM/864, La Patrie Créole, 6 juin 1919.

82. Idem.

83. Raphaël Barquissau, Enfance aux îles, Paris, À l'île des poètes, 1960, p. 45. D755.

84. Le Courrier Colonial, 17 juillet 1914, Archives de la République de Madagascar (ARM)

85. L'auteur de l'article mentionne le gouvernorat de John Hennessy. Il est vrai que cet Irlandais avait pris position après la révolte des Cipayes en Inde pour une politique d'ouverture envers les populations indiennes. C'est donc sûrement à ce titre qu'on le considère comme un " Hindou ". 
des terres appartenant aux Créoles. Ce conflit entre les Créoles de couleur et les migrants indiens entraine le risque "de voir disparaître de Maurice l'élément français ». La solution serait de favoriser la migration des FrancoMauriciens vers Madagascar pour qu'ils puissent former avec les Créoles de La Réunion une communauté partageant les mêmes valeurs et les mêmes intérêts. Il est vrai que «les Mauriciens ont formé, avec les Bourbonnais, le premier noyau de la colonisation à Madagascar ${ }^{86}$ ". Finalement, la peur de devenir dépendant de l'Inde rejoint celle exprimée par les Réunionnais lorsqu'il était question de rattacher politiquement leur île à Madagascar. Les termes ne sont certes pas les mêmes mais les sentiments éprouvés montrent quelques convergences.

Dans les discours, l'invasion des Indiens d'origine britannique dépasse le seul cadre de l'île Maurice. Par le biais d'une émigration mauricienne soutenue vers La Réunion, le danger concerne désormais les Réunionnais. La défiance que ces derniers éprouvent envers les Mauriciens ne concerne que les populations d'origine indienne vers lesquelles converge un faisceau de récriminations. Ces tensions sont surtout perceptibles lors des périodes de crise et inquiètent notamment le consul britannique de La Réunion qui déplore "une atmosphère de défiance à l'endroit des éléments étrangers à la Réunion, plus particulièrement des Mauriciens ${ }^{87}$ ". Les polémiques lancées par la presse sont nombreuses et débouchent inéluctablement sur un déferlement de haine xénophobe. Les réticences exprimées envers certains sujets mauriciens alimentent dans le même temps le mythe des îles sœurs qui se reconnaissent à la fois par leur commune origine et somme toute leur perte de repères identitaires. Finalement, cette identité indiaocéanique tant souhaitée se structure autour d'une double prise de conscience : la similarité de destin entre les élites créoles blanches des deux îles et leur singularité face à une mixité sociale imaginée de part et d'autre comme de plus en plus évidente. La racialisation croissante des rapports sociaux dans les mondes coloniaux indiaocéaniques explique en partie ce double mouvement de repli identitaire.

\section{CONCLUSION}

Initié avant la Grande Guerre par une poignée de Franco-Mauriciens nostalgiques d'une certaine France mythifiée puis dynamisé après 1918 par les conférences de paix promouvant le droit des peuples à disposer d'eux-mêmes, le mouvement de rétrocession de l'île Maurice à la France sert surtout de catalyseur à une réflexion identitaire. Dépassant très rapidement le seul cadre de l'île Maurice, les débats interpellent tout à la fois les puissances tutélaires françaises et britanniques et les sociétés coloniales dépendantes de leur hégémonie. L'écho de ces débats à La Réunion montre ainsi que

86. Le Courrier Colonial, 17 juillet 1914, op. cit.

87. ADR 4M209, Consul britannique au gouverneur de La Réunion, 14 décembre 1936. 
les empires ont été des expériences transculturelles, puis transnationales d'extraversion au cours desquelles des catégories populaires, mais aussi des élites politiquement dominantes, pouvaient se vivre à travers le prisme de la langue, des croyances, des valeurs ou du style de l'autre ${ }^{88}$.

Si ces interactions impériales se comprennent à l'aune d'une histoire coloniale partagée, elles mettent surtout en évidence les prémices d'une construction identitaire indiaocéanique dont le mythe des "îles sœurs " constitue l'avatar le plus probant. Ce mythe est notamment repris par Marius Leblond au lendemain de la Seconde Guerre Mondiale dans un ouvrage qui célèbre le caractère édénique de La Réunion et de Maurice, le génie de leurs habitants, surtout celui de «l'essaim des races blanches qui ont accueilli le kaléidoscope des races de couleur ${ }^{89}$ ". À partir des années 1960 , cet imaginaire se libère des conceptions racialistes initiales et inspire certains courants littéraires vantant les mérites d'un indianocéanisme qui, selon les termes de l'écrivain mauricien Camille de Rauville, se revendique d'un " nouvel humanisme au cœur de l'océan Indien ${ }^{90}$ ", ce que d'aucuns nomment aujourd'hui une "créolie indianocéaniste ${ }^{91}$ " ou encore une "indianocéanie des cultures ${ }^{92}$ ", structurée autour d'un héritage commun. Ce legs sert actuellement de matrice identitaire dans le cadre politique de la Commission de l'Océan Indien.

\author{
Pierre-Éric FAGEOL \\ $M C F$ Histoire \\ Université de La Réunion
}

88. Jean-François Bayard et Romain Bertrand, "De quel "legs colonial” parle-t-on ? ", Esprit, décembre 2006, p. 134-160.

89. Marius Leblond, Les Îles seurs ou le paradis retrouvé, La Réunion-Maurice, Paris, Éditions Alsatia, 1946, $251 \mathrm{p}$.

90. Voir Camille de Rauville, Anthologie de l'océan Indien, Tananarive, Éditions des cahiers de la Lémurie, 1956, 332 p. En 1961, cet auteur mauricien reprend le flambeau identitaire de son père Hervé de Rauville et définit le terme d'indianocéanisme comme « un nouvel humanisme au cœur de l'océan Indien ", une identité propre aux peuples riverains de l'océan Indien. En ce sens l'indianocéanisme se distingue de l'indiaocéanisme qui ne renvoie qu'à une volonté de localisation d'ordre géographique.

91. Voir Jean-Georges Prosper, "La créolie indianocéaniste ", Éthiopiques, n $53,1^{\text {er }}$ semestre 1991, non paginé.

92. "Les mille visages de l'indianocéanie ", Actes du colloque de la COI, Mahébourg, juin 2013. 